\title{
Reflexões sobre o processo de elaboração da Política de Preservação e Gestão de Acervos Culturais das Ciências e da Saúde da Casa de Oswaldo Cruz - Fiocruz.
}

\author{
Marcos José de Araújo Pinheiro 1 \\ Casa de Oswaldo Cruz / Fiocruz. \\ Carla Maria Teixeira Coelho \\ Casa de Oswaldo Cruz/ Fiocruz \\ Liene Wegner ${ }^{3}$ \\ Casa de Oswaldo Cruz/ Fiocruz
}

\section{RESUMO}

A Casa de Oswaldo Cruz ( $\mathrm{COC}$ ) é a unidade técnico-científica da Fundação Oswaldo Cruz (Fiocruz) responsável pela preservação e valorização da memória da instituição em seus campos de atuação. A equipe técnica da $\mathrm{COC}$ desenvolve ações de preservação de edifícios históricos, sítios urbanos, acervos arquivísticos, bibliográficos e museológicos que representam importante fonte de conhecimento. Como resultado da experiência e do amadurecimento da $\mathrm{COC}$ no que tange aos seus processos de trabalho e instrumentos de gestão e de planejamento institucional foi identificada a necessidade de elaboração de uma Política de Preservação e Gestão de Acervos.

O processo de elaboração desse documento teve início no começo de 2012, com a criação de um grupo de trabalho composto por integrantes dos diferentes departamentos e coordenado pela Vice-Diretoria de Informação e Patrimônio Cultural. $O$ documento base elaborado tem como objetivo definir princípios, diretrizes e orientações que devem reger as atividades de gestão e preservação dos acervos culturais das ciências e da saúde sob a guarda da COC.

O processo de construção coletiva do documento contempla sua avaliação por consultores externos ad hoc, um período de consulta interna e sua submissão ao Conselho Deliberativo da unidade para aprovação, e tem contribuído para uma 
maior integração entre as diferentes áreas envolvidas. O presente trabalho busca refletir sobre o processo de elaboração - ainda em andamento - da Política de Preservação e Gestão de Acervos Culturais das Ciências e da Saúde e discutir os futuros desdobramentos desse trabalho.

Palavras-chave: Política de preservação. Patrimônio cultural. Gestão (termo genérico, sugestão: Gestão de documentos ou Acervo - referência: Vocabulário controlado SIBi).

\section{Considerations on the drafting work of the Preservation and Management Policy for Cultural Collections in Science and Health of Casa de Oswaldo Cruz - Fiocruz}

The Casa de Oswaldo Cruz (COC) is responsible for the preservation and valorization of the Oswaldo Cruz Foundation (Fiocruz) institutional memory. Its professional team preserves historical buildings, urban sites, archival, library and museum collections which represent an important source of knowledge in the area of sciences and health in Brazil. The pertinence of a Preservation and Management Policy for Cultural Collections under the responsibility of $\mathrm{COC}$ is a result of the accumulated experience and matured understanding of the organization work processes and its management and planning tools.

A working group, which drew representatives from the areas responsible for the preservation of cultural collections, initiated the drafting of the policy in the beginning of 2012 under the coordination of the Vice-Directorship for Information and Cultural Heritage. The first draft of the document establishes principles, guidelines orientations that govern management and preservation actions applicable to the cultural collections in science and health under the custodianship of COC.

Its design was a collective effort that enjoyed the collaboration of ad hoc external consultants who evaluated the policy, as well as a period of internal hearing, and the submission to the COC's Deliberative Council for approval. This process has 
contributed for a larger collaboration between the different organizational structures responsible for the preservation of the cultural collections.

This paper attempts to consider the still on working drafting process of the Preservation and Management Policy for Cultural Collections in Science and Health and its outcomes.

Keywords: Preservation Policy. Cultural heritage. Management.

\section{INTRODUÇÃO}

Criada em 1900, como Instituto Soroterápico Federal, a Fundação Oswaldo Cruz (Fiocruz), instituição pública de pesquisa ligada ao Ministério da Saúde, sediada no Rio de Janeiro, é responsável pela preservação de importante acervo relacionado ao patrimônio cultural da saúde. Grande parte desse acervo foi gerada ao longo de mais de um século pelos processos de trabalho da própria instituição, que tem como missão a promoção da saúde e o desenvolvimento social.

A Casa de Oswaldo Cruz (COC), criada em 1985, é a unidade técnico-científica da Fiocruz responsável pela preservação da memória da instituição e pelas atividades de pesquisa, ensino, documentação e divulgação da história da saúde pública e das ciências biomédicas no Brasil. Atualmente, a equipe técnica da $\mathrm{COC}$ atua na preservação de edifícios históricos, sítios urbanos, acervos arquivísticos, bibliográficos e museológicos que representam importante fonte de conhecimento.

Visando garantir a preservação dos diferentes tipos de acervos sob sua responsabilidade a estrutura da $\mathrm{COC}$ foi organizada, ao longo de sua existência, em subestruturas com atuações específicas: Departamento de Arquivo e Documentação (criado em 1986); Departamento de Patrimônio Histórico (1989); Biblioteca de História das Ciências e da Saúde (1991); Museu da Vida (1994); e Biblioteca de Educação e Divulgação Científica (1999). 
O Departamento de Arquivo e Documentação é responsável pelo acervo arquivístico da unidade, reunindo fundos e coleções de documentos institucionais e pessoais cujos parâmetros temáticos são as ciências da vida - que envolvem as ciências da saúde, as ciências biomédicas, as ciências biológicas - e as ciências humanas e sociais alinhadas à missão institucional.

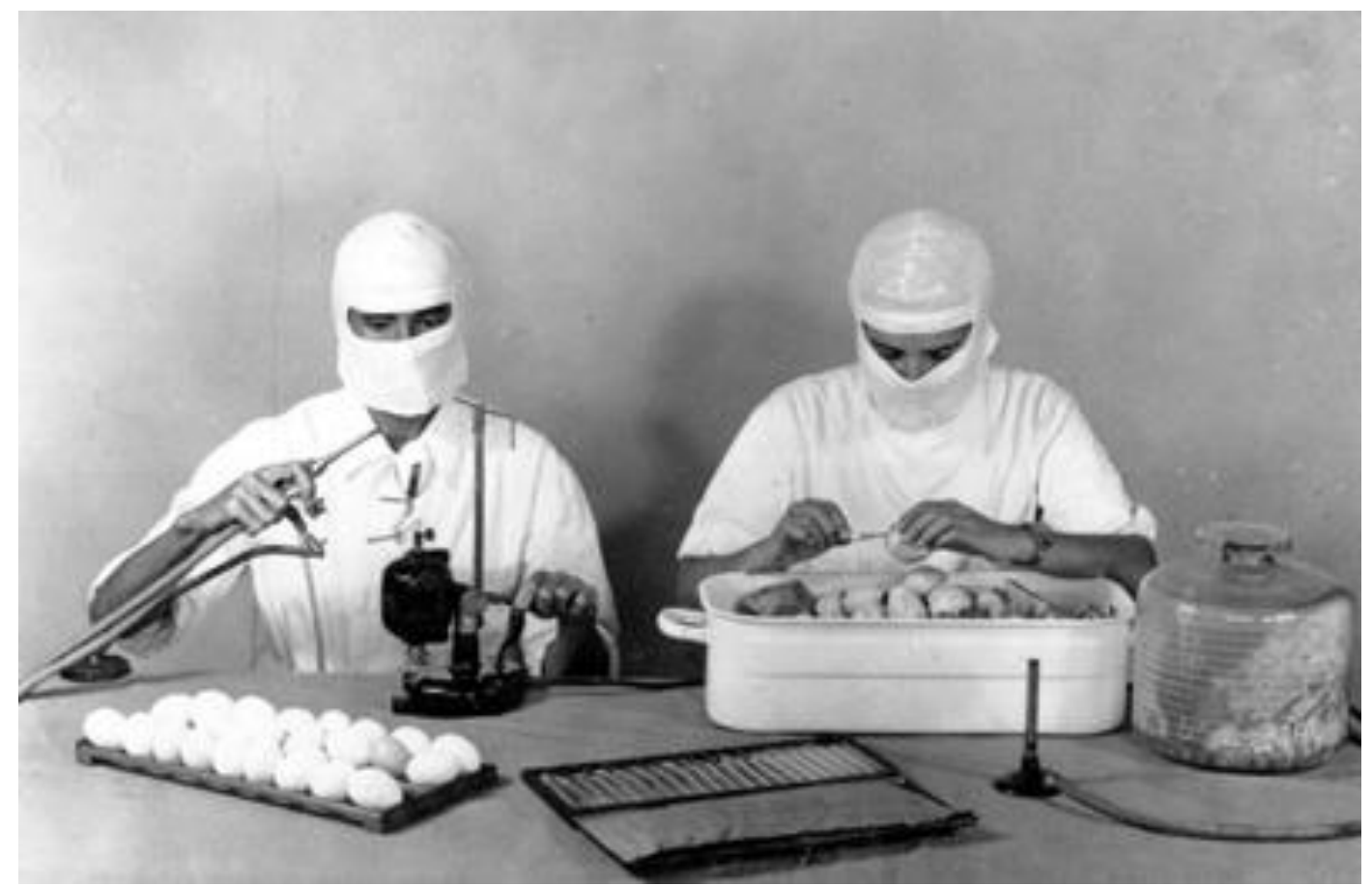

FIGURA 1: Fotografia da produção de vacina contra a febre amarela no laboratório construído pela Fundação Rockefeller no Instituto Oswaldo Cruz, 1943. Fundo Fundação Rockefeller/Acervo COC.

Fazem parte do acervo documentos textuais, iconográficos, cartográficos, sonoros e filmográficos que abrangem o período compreendido entre o início do século XIX e os dias atuais. Conta com uma equipe formada, predominantemente, por arquivistas e historiadores.

O Departamento de Patrimônio Histórico atua na preservação do acervo arquitetônico, urbanístico e arqueológico sob gestão da Fiocruz 


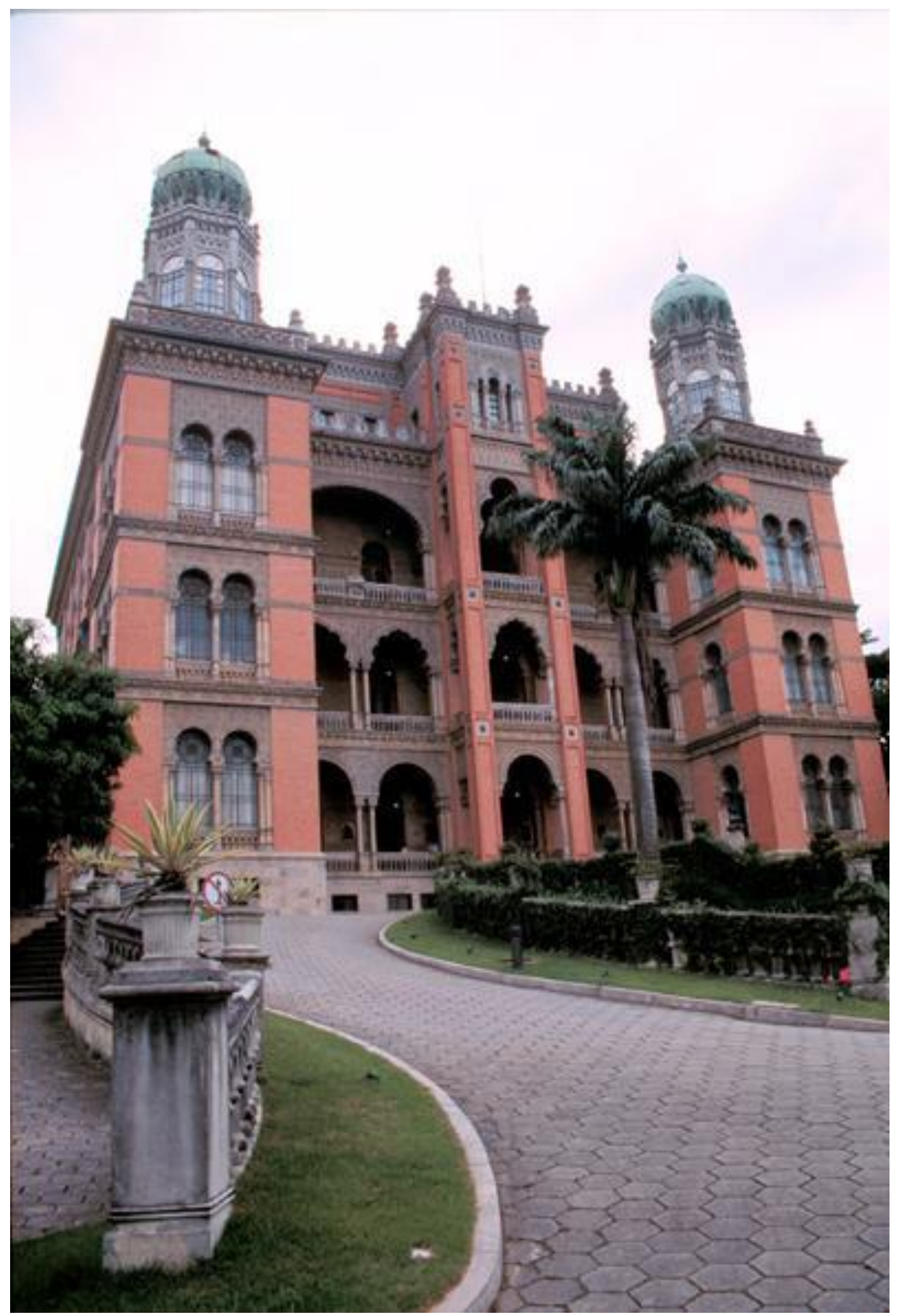

FIGURA 2: Pavilhão Mourisco. Edificação que compõe o Núcleo Arquitetônico Histórico de Manguinhos. Foto: Peter Illicciev.

Atualmente compõem esse acervo edifícios históricos, sítios urbanos e vestígios arqueológicos localizados no campus Fiocruz Manguinhos, no campus Fiocruz Mata Atlântica (ambos na cidade do Rio de Janeiro) e no conjunto edificado do Palácio 
Itaboraí (em Petrópolis, no estado do Rio de Janeiro). Sua equipe é constituída majoritariamente por arquitetos e engenheiros.

O Museu da Vida (MV) é responsável pela preservação de acervo museológico classificado na área da ciência e tecnologia em saúde, ligado principalmente à história institucional. 


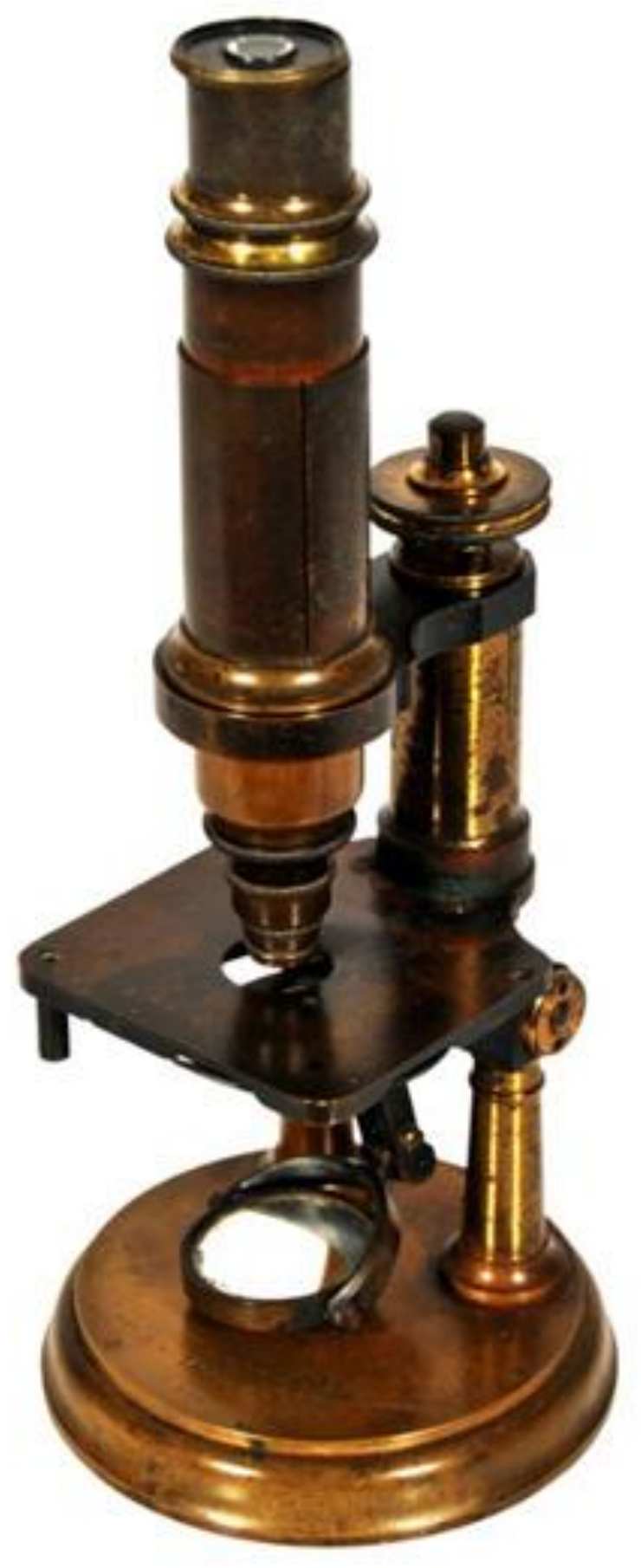

FIGURA 3: Microscópio de estudante. Acervo Reserva Técnica/Museu da Vida. Foto: Vinicius Pequeno e Roberto Jesus Oscar.

O acervo é formado por instrumentos e equipamentos de laboratório, materiais e maquinário utilizados na produção de medicamentos e vacinas, instrumentos 
médicos, mobiliário, indumentária, objetos pessoais de cientistas da instituição e uma pinacoteca, e abrange o período compreendido entre meados do século XIX e a atualidade. A equipe da Reserva Técnica do MV é formada por museólogos e historiadores.

O Museu da Vida é responsável ainda pela Biblioteca de Educação e Divulgação Científica, cujo acervo abrange as áreas de divulgação científica, educação, museologia, ciências da vida, saúde e literatura infantojuvenil, e é formado por obras de referência, monografias, teses, dissertações, folhetos, jogos e material audiovisual.

A Biblioteca de História das Ciências e da Saúde reúne acervo bibliográfico especializado em História da Medicina, História da Saúde Pública, História, Sociologia e Filosofia da Ciência. O acervo é constituído por monografias, obras de referência, teses, dissertações, folhetos e material eletrônico, com abrangência temporal que vai do século XVII aos dias atuais. Os profissionais responsáveis por ambas as bibliotecas possuem formação em Biblioteconomia.

Cada um dos departamentos e setores mencionados possui equipe técnica própria que atua na preservação e gestão desses acervos, e ainda colabora com ações de outras unidades da Fiocruz ${ }^{4}$. Ao longo dos seus 25 anos, a $\mathrm{COC}$ vem acompanhando o debate nacional e internacional acerca da preservação do patrimônio cultural. As ações realizadas pela COC nos diferentes tipos de acervo sob a sua guarda refletem o diálogo com esse debate, matizado pelas leituras disciplinares das diversas formações que compõem o quadro de profissionais responsáveis por esses acervos na unidade e pelas lógicas institucionais de gestão e tutela de tais acervos. Em sintonia com as discussões do campo da preservação, nos últimos anos a COC vem buscando investir em ações preventivas para garantir a conservação desses acervos, fomentando a interdisciplinaridade entre as equipes de seus diferentes departamentos e uma abordagem mais sistêmica sobre a gestão dos acervos.

A partir dos anos de 1980 assistimos a um aprimoramento do planejamento a médio e longo prazo nas instituições públicas. Planos plurianuais passaram a estabelecer 
as metas estratégicas e os resultados institucionais esperados e a orientar os investimentos e o aporte de recursos para a execução das ações necessárias. Na Fiocruz, a elaboração do Plano Quadrienal é um processo coletivo e passa pela construção de consensos intra e entre as diversas unidades que compõem a instituição. O fortalecimento da área de planejamento e gestão institucional tem também contribuído para a construção de uma linguagem comum que facilite a compreensão e a incorporação dos objetivos institucionais.

Como resultado da experiência e do amadurecimento da COC, no que tange aos seus processos de trabalho e instrumentos de gestão e de planejamento institucional, foi identificada a necessidade de elaboração de uma Política de Preservação e Gestão de Acervos que estabelecesse princípios teóricos e diretrizes gerais a serem seguidos por todas as áreas. A COC propôs a inclusão de item sobre o desenvolvimento de políticas de preservação para os acervos institucionais no Plano Quadrienal da Fiocruz 2011-2014, e, em consonância com sua missão ${ }^{5}$, incorporou essa ação também ao Plano Quadrienal da própria unidade para o mesmo período.

\section{MÉTODO E PROCESSO}

O processo de elaboração desse documento teve início na Câmara Técnica de Informação e Documentação da COC e de sua Subcâmara de Patrimônio Cultural. No começo de 2012 foi instituído pela direção da unidade um grupo de trabalho composto por integrantes dos diferentes departamentos e coordenado pela ViceDiretoria de Informação e Patrimônio Cultural, e que foi denominado GT Política de Preservação6.

A partir das discussões iniciadas na Subcâmara de Patrimônio Cultural, e tendo como horizonte temporal o prazo inicial de sete meses ${ }^{7}$ dado ao GT para elaboração da política, organizou-se uma agenda de reuniões semanais, um cronograma de validação do documento e a divisão do trabalho em três fases: a) levantamento, apresentação e discussão de textos de referência; b) definição da estrutura do documento; c) redação do texto. 
Respondendo ao princípio da transparência interna, o GT disponibilizou no portal corporativo da Casa de Oswaldo Cruz, desde o início dos trabalhos, todas as atas de reunião, documentos de referência utilizados, apresentações e cronograma de reuniões como estratégia para o acompanhamento de suas atividades por parte do conjunto de trabalhadores da COC.

$\mathrm{Na}$ primeira fase dos trabalhos foram levantadas referências bibliográficas sobre 0 tema, incluindo exemplos de políticas elaboradas por instituições brasileiras e estrangeiras e materiais já produzidos pelos próprios departamentos da unidade (manuais, planos e normas). Foram pesquisadas referências que abrangessem todos os tipos de acervos sob a guarda da $\mathrm{COC}$ e cada membro se encarregou da leitura e da apresentação de um número de textos. Evitou-se, na medida do possível, designar aos membros do GT a apresentação de textos relacionados à sua área específica de atuação, incentivando, assim, a aproximação com conceitos e processos de outras áreas.

Uma das dificuldades iniciais encontradas por esse GT decorreu do processo histórico de como as áreas se organizaram e constituíram os processos de preservação e gestão das diferentes tipologias de acervos. De fato, o que ocorreu na unidade não se configura como uma particularidade, e sim espelha uma realidade nacional que pode ser evidenciada pela autonomia relativa entre as instâncias de regulamentação e governança que atuam na preservação do patrimônio cultural brasileiro. Apenas para exemplificar, no caso da Fiocruz, onde há acervos arquivísticos, arquitetônicos e urbanísticos, bibliográficos, museológicos e biológicos, os órgãos responsáveis na esfera federal pela regulamentação e tutela de cada tipologia de acervo estão vinculados a diferentes ministérios, tais como o Ministério da Cultura, o Ministério da Justiça, o Ministério do Meio Ambiente; e ainda o Ministério da Saúde e o Ministério da Ciência e Tecnologia, pelos aspectos específicos dos acervos institucionais.

Os efeitos desta setorização não podem ser compreendidos apenas com base no contexto político-administrativo, mas principalmente pelos aspectos históricos e culturais de como se construiu a noção nacional de patrimônio cultural. Internamente, tanto na Fiocruz como na $\mathrm{COC}$, essa diferenciação e a pouca 
articulação entre as áreas resultaram em baixo nível de cooperação e em conceituações distintas de terminologia, o que implicou numa maior dificuldade de comunicação e na necessidade de serem estabelecidas definições conceituais de consenso com base na bibliografia, nas normativas usuais para cada tipo de acervo e nas distintas culturas de trabalho.

As leituras buscaram também identificar os tópicos abordados nos textos de políticas a fim de subsidiar as discussões da segunda fase dos trabalhos, que tratou de estabelecer a estrutura do documento. Outro desafio enfrentado foi a diversidade dos modelos de política de preservação de instituições brasileiras e estrangeiras encontrados pelo GT na web. A pesquisa realizada pelo GT focou, num primeiro plano, documentos que tratassem de orientações e definições teóricas do objetivo, do significado e de como deve ser uma política de preservação. E num segundo plano, buscou identificar políticas que contemplassem ao menos um dos tipos de acervos que são objetos de nossa política, ou seja, arquivístico, arquitetônico e urbanístico, bibliográfico e museológico.

Tanto 0 plano da orientação e definição teórica quanto 0 das políticas disponibilizadas pelas instituições na web demonstravam uma não padronização no modo como as instituições se apresentavam em seus portais e sítios. Algumas políticas eram extremamente sucintas e pouco esclarecedoras; outras eram extensas, muito detalhadas, se aproximavam mais de manuais de procedimentos de preservação patrimonial, e acabavam por diluir no corpo do texto as diretrizes políticas. Por outro lado, o que se identificou foi o predomínio de políticas para acervos bibliográficos e arquivísticos, algumas políticas para acervos museológicos, e em relação aos acervos arquitetônicos e urbanísticos, o que mais havia eram planos de preservação. Políticas que abarcassem diferentes tipologias de acervos foram poucas.

Uma referência fundamental para o desenvolvimento do trabalho foi o documento Building Blocks for a Preservation Policy, de Mirjam M. Foot (2001). Nesta publicação da British Library a autora conceitua de modo claro a diferença central entre políticas e estratégias. De acordo com Foot (2001), uma política de preservação é um plano de ação que deve considerar: o que deve ser preservado; 
por que deve ser preservado; para que deve ser preservado; e por quanto tempo. Já uma estratégia de preservação deve considerar: como fazer; em que ordem/prioridade, ou melhor, quando. A autora destaca ainda a importância desse tipo de iniciativa em relação à divulgação e monitoramento das ações:

Organizações e instituições, particularmente seus dirigentes e financiadores, mas igualmente seus usuários, esperam uma política como sinal de responsabilidade e como uma expressão tangível de intenção, assim como algo que pode ser monitorado. Uma política pode explicar aos usuários por que certas ações são ou não são tomadas. Ela define as responsabilidades de todos os envolvidos, da equipe de funcionários aos usuários (FOOT, 2001, p.1-2, tradução nossa).

Com base no modelo de gestão democrática participativa vigente na Fiocruz, somado às sugestões encontradas nos textos de referência, o GT definiu o processo de validação e de aprovação do documento da política que envolveu: a apresentação e discussão de uma primeira versão em reuniões com a Câmara Técnica de Informação e Documentos e Subcâmara de Patrimônio Cultural da COC e com o Conselho Deliberativo (CD) da unidade; a avaliação por consultores externos ad hoc e a consulta interna, com a disponibilização do documento no portal corporativo e a discussão do mesmo em apresentações na unidade. A submissão final ao CD da unidade concluirá a fase de aprovação.

Complementando o processo de validação, os representantes departamentais membros do GT foram orientados a relatar as atividades do grupo nos fóruns departamentais e colher as eventuais contribuições ao documento ao longo de todo o período de elaboração do documento.

A terceira fase dos trabalhos envolveu a redação do texto da política a partir da estrutura definida. Foi estabelecida uma relatoria para a organização e a edição dos textos. Teceremos na seção seguinte algumas considerações sobre o documento resultante ${ }^{8}$.

\section{RESULTADOS PRELIMINARES}


A estrutura proposta para o documento base ${ }^{9}$ - organizado em um bloco principal e dois anexos - buscou apresentar de maneira clara os diferentes acervos sob responsabilidade da unidade e definir os princípios teóricos que deverão guiar as ações de preservação referentes a eles.

A etapa de definição de conceitos e princípios teóricos exigiu o estabelecimento de consenso entre as diferentes áreas. Tendo em vista o objetivo do grupo de redigir uma política que esteja alinhada com os princípios internacionalmente consagrados no campo da preservação do patrimônio cultural, foram utilizados como referências documentos elaborados por instituições como United Nations Educational Scientific and Cultural Organization (Unesco), International Council on Monuments and Sites (Icomos) e International Council of Museums (Icom). Os princípios, assumidos como declarações de valor, estão refletidos nas diretrizes e deverão permear as ações de gestão e de preservação dos acervos ${ }^{10}$.

O documento estabelece a necessidade de revisão da política em intervalos máximos de quatro anos. Dessa forma, espera-se garantir a atualização periódica desses conceitos e diretrizes seguindo os princípios internacionais e as orientações dos órgãos de tutela e preservação ${ }^{11}$.

São definidos ainda seis programas de preservação e gestão comuns aos acervos: Programa de Incorporação; Programa de Tratamento Técnico; Programa de Conservação e Restauração; Programa de Segurança; Programa de Acesso, Empréstimo e Reprodução; e Programa de Difusão Cultural. O enunciado desses programas, agrupados em um dos anexos do documento, estabelece os conteúdos e objetivos. O desenvolvimento desses itens faz parte de uma etapa subsequente do trabalho e revela o desafio de agrupar em programas comuns questões que até então vêm sendo tratadas de maneira isolada pelas áreas.

\section{CONSIDERAÇÕES FINAIS}

A elaboração do documento base da Política de Preservação e Gestão de Acervos Culturais das Ciências e da Saúde da Casa de Oswaldo Cruz é resultado de um longo processo que antecede a instalação do grupo de trabalho responsável por 
essa tarefa. É fruto do aprimoramento dos processos de trabalho e dos instrumentos de planejamento e gestão institucional da $\mathrm{COC}$ e da Fiocruz em paralelo com a ampliação do conceito de patrimônio cultural e da formação do campo da preservação do patrimônio enquanto área de conhecimento.

A Casa de Oswaldo Cruz tem feito esforços para estimular uma abordagem mais sistêmica na gestão dos acervos sob sua guarda, para criar mecanismos que facilitem a aproximação entre as áreas responsáveis por tais acervos e que promovam a formação de recursos humanos e a pesquisa na área de preservação do patrimônio. A proposta de criação de um programa como o Complexo de Preservação e Difusão de Acervos Científicos da Saúde que promova uma gestão integrada dos diferentes acervos culturais da Fiocruz por parte das distintas unidades responsáveis busca ampliar as parcerias internas e reforçar a área de preservação do patrimônio cultural na agenda institucional. A Subcâmara de Patrimônio Cultural, criada como instância consultiva e de assessoramento à direção da COC, passou a reunir profissionais da unidade que trabalham na área de preservação e a suscitar o debate de temas afetos a esse campo. Em iniciativa recente, foi estabelecido como critério para seleção de projetos de pesquisa a serem fomentados por edital interno o envolvimento de membros de mais um departamento da unidade na equipe do projeto. O Curso de Especialização em Preservação e Gestão do Patrimônio Cultural das Ciências e da Saúde da COC, implantado em 2010, reúne em seu corpo docente profissionais que atuam em diversas áreas da unidade e em outras instituições, e tem como objetivo formar especialistas para a prática profissional e pesquisa acadêmica com ênfase no planejamento e execução de ações voltadas para a preservação e gestão do patrimônio arquitetônico e documental das ciências e da saúde.

Todas essas iniciativas convergem para a implantação do documento base da Política de Preservação e Gestão de Acervos Culturais das Ciências e da Saúde da Casa de Oswaldo Cruz. Ainda assim, restam muitos desafios a serem superados. Entre eles podemos apontar a definição de novas estratégias para estimular a integração das equipes e garantir que as ações de preservação nas diferentes áreas sigam os mesmos princípios teóricos; e a implementação de instrumentos para monitorar a eficácia da política proposta. 


\section{Notas}

1 Engenheiro eletricista (PUC-RJ), Doutor em engenharia de produção (COPPE/ UFRJ), Vice-Diretor de Informação e Patrimônio Cultural da Casa de Oswaldo Cruz / Fiocruz.

E-mail: mjap@coc.fiocruz.br

2 Arquiteta e urbanista (FAU / UFRJ), Mestre em arquitetura (PROARQ / UFRJ), arquiteta do Departamento de Patrimônio Histórico da Casa de Oswaldo Cruz/ Fiocruz.

E-mail: carlacoelho@coc.fiocruz.br

3 Graduada em relações internacionais (UnB), Mestre em política e gestão de C\&T\&I em saúde (ENSP / Fiocruz), responsável pela Assistência Técnica de Cooperação da Casa de Oswaldo Cruz / Fiocruz.

E-mail: Iwegner@coc.fiocruz.br

4 Além dos acervos citados, a Fiocruz possui ainda outros acervos bibliográficos - com destaque para a coleção de obras raras - e importantes coleções biológicas que começaram a ser organizadas no início do século XX, como resultado das expedições científicas realizadas pelos pesquisadores da instituição.

5 A missão atual da Casa de Oswaldo Cruz é "Produzir e disseminar o conhecimento histórico da saúde e das ciências biomédicas; preservar e valorizar o patrimônio cultural da saúde; educar em seus campos de atuação e divulgar ciência e tecnologia em saúde, de forma a contribuir para o desenvolvimento científico, cultural e social" (CASA DE OSWALDO CRUZ, 2011).

6 O GT é composto por profissionais com formação em arquitetura e urbanismo, arquivologia, biblioteconomia, engenharia, história, museologia e relações internacionais, e que atuam na preservação dos acervos culturais sob a guarda da COC.

70 prazo dos trabalhos foi estendido para melhor acomodar o tempo de resposta dos consultores e o período de consulta interna, além da agenda institucional. 
80 texto base da Política de preservação e gestão de acervos culturais das ciências e da saúde está disponível para consulta através do Portal da Casa de Oswaldo Cruz (http://www.coc.fiocruz.br/patrimonio/images/stories/PDFs/politica_preservacao_gestao_acervos_coc. pdf).

9 O sumário do documento foi estruturado da seguinte forma: Introdução; Definições; Objetivo; Princípios; Diretrizes; Programas de Preservação e Gestão; Responsabilidades; Normas; Financiamento; Revisão; Política de Preservação e Gestão do Acervo Arquitetônico, Urbanístico e Arqueológico; Política de Preservação e Gestão do Acervo Arquivístico; Política de Preservação e Gestão do Acervo Bibliográfico; Política de Preservação e Gestão do Acervo Museológico; Referências; Anexo I - Programas de Preservação e Gestão; Anexo II - Normas.

10 A política assume o patrimônio cultural como representação da memória e da identidade de um grupo, como fruto da ação humana, como fonte de pesquisa e passível de atribuição a múltiplos valores. Assume também que na relação do patrimônio cultural com a sociedade devem ser adotados como princípios o compromisso com as gerações futuras e com populações locais; com a cidadania plena; a solidariedade; o respeito à diversidade étnica e sociocultural; o comprometimento socioambiental; a democratização do conhecimento; a educação como processo emancipatório; e a ética e transparência. Em consonância com estes princípios, assumem-se como orientações estruturantes a conservação preventiva, o gerenciamento de riscos, a conservação integrada, a preservação sustentável e ainda a educação patrimonial e a pesquisa e desenvolvimento em preservação de acervos (CASA DE OSWALDO CRUZ, 2013).

11 Atualmente as instituições que exercem algum tipo de tutela sobre os acervos da COC são: United Nations Educational Scientific and Cultural Organization (Unesco), (), Instituto do Patrimônio Histórico e Artístico Nacional (Iphan), Instituto Estadual do Patrimônio Cultural (Inepac) e Prefeitura da Cidade do Rio de Janeiro.

\section{REFERÊNCIAS}

CHAPMAN, Patricia. Guidelines on preservation and conservation policies in the archives and libraries heritage. Paris: Unesco, 1990.

FOOT, M.M. Building blocks for a preservation policy. Londres: The British Library, 2001. Disponível em: <http://www.bl.uk/blpac/pdf/blocks.pdf>. Acesso em: 22 fev. 2011. 
FUNDAÇÃO OSWALDO CRUZ. CASA DE OSWALDO CRUZ. Manual da organização. Portal da Casa de Oswaldo Cruz, $2007 . \quad$ Disponível em: <http://www.coc.fiocruz.br/institucional/images/stories/manual organizacao coc.pdf $>$. Acesso em: 13 fev. 2013. Plano quadrienal 2011-2014. Portal da Casa de Oswaldo Cruz, 2011. Disponível em:<http://www.coc.fiocruz.br/institucional/images/stories/plano quadrienal coc.pdf >. Acesso em: 13 fev. 2013.

FUNDAÇÃO OSWALDO CRUZ. Plano quadrienal 2011-2014. In: FIOCRUZ. VI Congresso Interno da Fiocruz: Relatório final. Portal $2010 . \quad$ Disponível em: <http://www.fiocruz.br/congressointerno/media/relatorio final ultima versao.pdf >. Acesso em: 13 fev. 2013.

FUNDAÇÃO OSWALDO CRUZ. CASA DE OSWALDO CRUZ. Política de preservação e gestão de acervos culturais das ciências e da saúde. Rio de Janeiro: Fiocruz/COC, 2013. Disponível em: < http://www.coc.fiocruz.br/patrimonio/images/stories/PDFs/politica_preservacao_gestao_acervos_coc.pdf>. Acesso em: 19 abr. 2013.

MUSEU DE ASTRONOMIA E CIÊNCIAS AFINS. Política de preservação de acervos institucionais. Rio de Janeiro: MAST / CNPq, 1995. 